\title{
TELEOPERADED BACKHOE EXCAVATOR WITH HAPTIC CONTROL
}

\author{
A. Barrientos, O. Luengo, A. Mora \\ DISAM. Universidad Politecnica de Madrid. C/Jose Gutierrez Abascal, 2. \\ E-28006, Madrid, Spain \\ (barrientos, oluengo,amora)@disam.upm.es.Tel + 34 913363061.Fax +34913363010
}

\begin{abstract}
Teleoperation has demonstrated its utility to work in hazardous and nonstructured environments. Nuclear and spatial applications have used telerobotics for remote manipulation in assembly or inspection tasks. In the near future, new tasks related with soil movements and other construction and civil engineering activities will be necessary in nuclear and spatial applications. In this paper a teleoperated backhoe excavator, remotely operated using visual and force feedback to the operator is presented. Different force feedback strategies and kinematic master-slave relation are discussed.
\end{abstract}

Keywords: Teleoperation, Bilateral control, Haptic devices

\section{INTRODUCTION}

Nowadays the intensive use of robots in hazardous environments, such as space or nuclear power plants, has motivated the use of teleoperation techniques due to the complexity of certain tasks.

The teleoperation has become a very useful technique in fields where a fast decision capacity is needed. Instead of using a program that takes the decision, and, in fact, close the control loop, it is the human brain (more specifically its cognitive and sensorial systems) what it is used. Of course, the best solution would be the whole automation of the task, but the state of the art in AI and/or the limited budget do not allow it. Nowadays, there are several research groups focused in the development and improvement of teleoperated systems for using them in a wide spectrum of applications (surgery, waste handling, surveillance, cleaning, underwater activities, defence, etc.). In the case of excavation the teleoperation allows the use of trained operators that will increase the productivity and the safety of the system.

The main goal of our study is the teleoperation of a backhoe excavator. In recent publications it has been shown that this tasks is one of the most challenging topics in the robotics research. These studies show its importance in some non-standard tasks like radioactive and waste handling and removal, nuclear power plants demolition, demining and extraterrestrial construction.[6]
There are some degrees of automation in the different projects that have been carried out in recent years. In some developments the primary goal is achieve just the remote control of the earth-moving machine, while in other ones the requirement is the full automation of the process. Some researchers have focused in theoretical aspects, while others present practical solutions to the problems they have found. In the next lines a brief description of some projects related with the work developed at DISAM will be given.

The University of Lancaster has been developing an excavator system to test new control algorithms (mainly focused on fuzzy logic and heuristics) for a teleoperated system. It implements force feedback through velocity measurements (what they call software force feedback). [2]. They affirm the velocity error vector is a good predictor of the force the excavator exerts. There is no analysis about bilateral control architectures, and possible stability problems.

The Fujikura company has developed an hybrid system composed of an excavator arm and a robotic manipulator, both of them mounted on a small caterpillar. They use just one joystick to control the four axis of the excavator as well as the manipulator. There is no information about the type of bilateral control that is used. [4]

The University of Purdue has focused its research in theoretical problems such as bucket path planning 
and dynamic model of the machinery. It has been developed a compliance control called cognitive force control, to avoid the presence of too large forces. There is no practical application documented (the results that are showed are SIMULINK simulations). [9]

The University of British Columbia has focused its efforts in the development of an impedance controller for a teleoperated excavator. The first results are showed with just one degree of freedom. They have developed a simulator that implements a theoretical soil model to feedback the force he would feel in the real site. [3]

Carnegie Mellon University is developing a pure autonomous excavator. The automation covers the whole excavation process, from the bucket path planning to the material dump on a truck. There is a high level planner that decides where and how to dig, as well as a local optimal planner that provides the shape of each dig. By far, it is the most advanced project the authors have found in this research area. [8]

DISAM, a research department of the Universidad Politecnica de Madrid, with funds provided by the CICYT (a joint institute of the Education and Industry Departments of the Spanish Government) is developing methods and algorithms to teleoperate a backhoe excavator with different bilateral control architectures with force feedback. [1]

The purposc of the project is to provide to the operator an easy way to work too. If the task to accomplish is too repetitive, although it was simple, it can produce that the operator feels tired, so the efficiency and the safety fall down. To avoid it, artificial intelligence techniques have been used to provide some kind of supervisory control. Basically, it consists in the automation of some repetitive tasks such as digging holes and trenches. It has been accomplished through learning from the opcrator the way he does the task. In order to achieve good results with the learning, fuzzy logic and neural networks have been used.

\section{DESCRIPTION OF THE SYSTEM.}

The core of the system is the backhoe excavator (see figure 1). Due to the intention of using commercial devices, it was needed to make some modifications to a standard excavator. They consisted in the implementation of a new hydraulic circuit and the addition of position sensors (rotational encoders) and pressure transducers. The hydraulic circuit is commanded through the use of proportional valves that provides good dynamic response and high quality control.

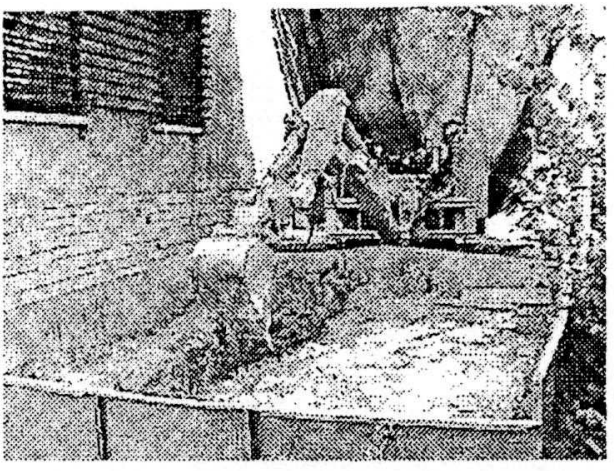

Figure 1. Backhoe excavator

A multi-axis controller board makes the control of the excavator. This board is responsible for the local loops, electrical inputs and outputs (including $\mathrm{A} / \mathrm{D}$ and $\mathrm{D} / \mathrm{A}$ converters). Inside the board, a PID algorithm is implemented. This algorithm is used in an adaptive way to control de movement of the excavator.

The computer hardware platform that has been used is PC with Pentium processor and with a National servomotion board, this card is used to control the proportional valves and to close the local loop. In addition, a data acquisition board with A/D converters is used to read the pressure transducers. (see figure 3).

A master device is incorporated to carry out the teleoperation of the excavator. It provides the force reflection capacity to the system, which means a better performance in telcoperated tasks and better knowledge of the environment from the operator point of view. It has some drawbacks too: it can produce unstability in the system, which requires a complex control strategy. Different master devices has been tested (see figures 2, 6 and 7)

Additionally, a video system has been incorporated to obtain a visual feedback to the operator.

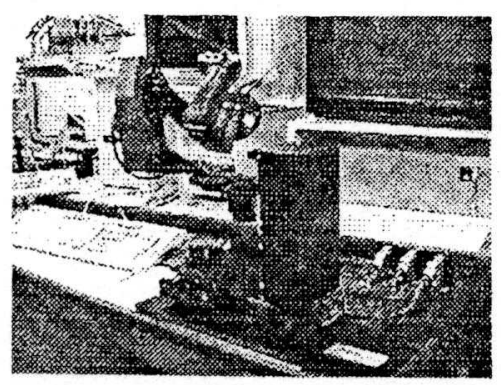

Figure 2. Cybernet Hand Controller 


\section{THE CONTROL SYSTEM}

As it has been written above, the hydraulic drives have a non-linear behaviour. The dynamics of the whole system (structure of the excavator plus hydraulic drives) is highly non-linear what means a complex control. At the low-level control, there is a reactive strategy in order to deal with unexpected facts. In fact, the main function of this layer is to avoid the contact with important buried objects such as pipes. It has been developed an algorithm of buried obstacles detection based upon pressure measurements to deal with this important aspect of the control system.

In the axis control level, it has been used an adaptive (gain scheduling) scheme of the parameters of an advanced PID algorithm (implemented in the control card). The parameters are changed in a continuous way to guarantee smoothness and good dynamic response. The change follows a set of rules based upon the configuration of the excavator arm, the constraints (mainly soil parameters, or reach), and the human commands (the latest only in teleoperation mode).

In the teleoperation mode, the chosen command option has been the sending of velocity commands during the bucket positioning, and position commands while digging. This solution provides good accuracy avoiding the usc of high transformation constants (from master device space to excavator space) that would producc undesired oscillations and lack of precision. On the other side, the chosen control strategy during teleoperation is the well know force - position scheme of bilateral control (see figure 4) with the variations proposed by Yosikawa in [10]. It takes into account not only the force at the tip of the bucket, but the position error in the calculation of the force reflected to the human operator. This feature provides more information about the bucket position, and avoids some problems with unstabilities due to some movements of the operator's hand (the force reflected in the master device makes more difficult the transmission of small involuntary movements to the slave). [6]

\section{REMOTE HAPTIC OPERATION}

As it has been writton above a master device is incorporated to carry out the teleoperation of the excavator. Scientists, engineers and psychologists have done a lot of studics related with teleoperation, and most of them agree in one point: in order to achieve good results in teleoperated systems force reflection must be used. Another question is how to implement it, but the fact is it is needed.

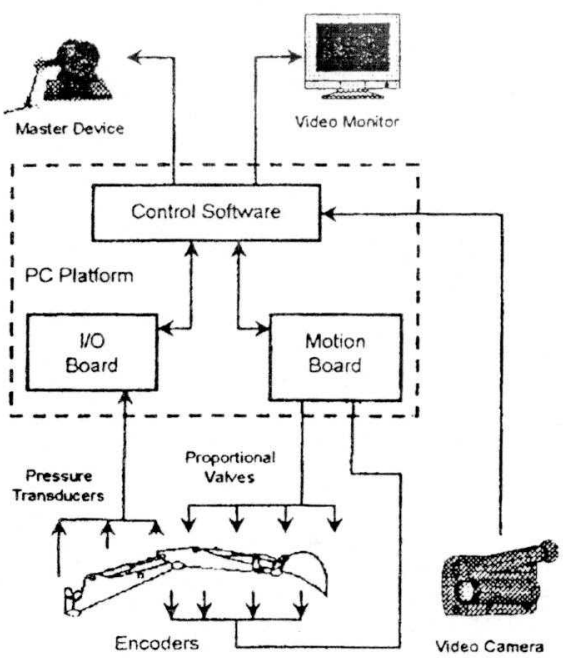

Figure 3. Control System

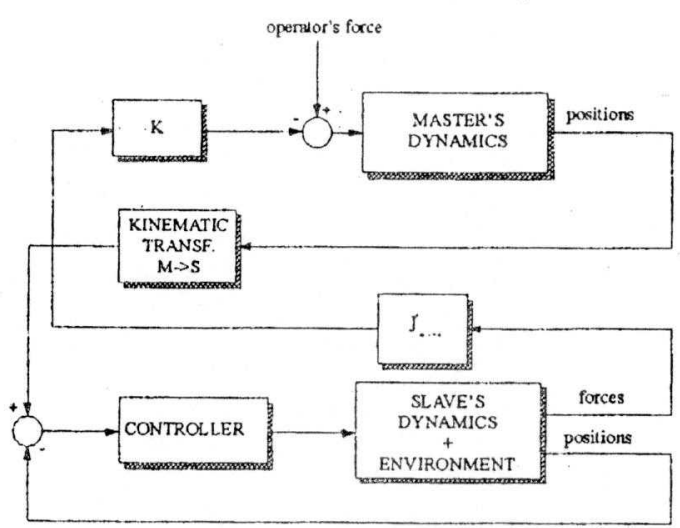

Figure 4. Force - position bilateral control

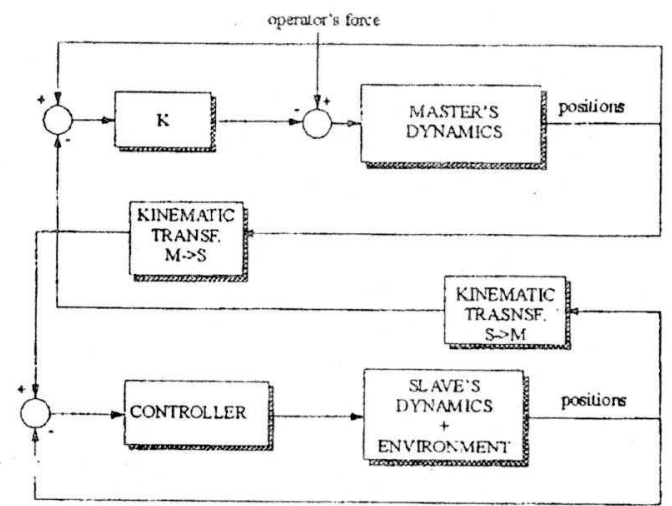

Figure 5. Position - position bilateral control

The master devices used in this project (see figures 2, 6 and 7) provide six degrees of frcedom; three translations $(\mathrm{x}, \mathrm{y}$ and $\mathrm{z})$ and three rotations (roll, 
pitch and yaw). For the purpose of command an excavator, only 4 are needed: three translations, and one rotation, or two translations and two rotations. In the first configuration, the three translations are used for positioning the bucket coordinate system and the rotation for spinning the bucket. In the second one the first rotation swings boom, stick and bucket, then there is a planar movement to place the bucket (two translations), and the last rotation for spinning the bucket. The operator in the manmachine interface chooses the configurations.

Three different master devices were used to carry out the teleoperation. At first, it has been used a Cybernet Hand Controller (Figure 2), it is a cartesian device with six degrces of freedom, three translations for position ( $x, y$ and $z$ ), and three rotations for orientation (roll, pitch and yaw), additionally it provides a force reflection in its six axis. It's a commercial device with serial interface with the computer. Due to its cartesian kinematics its use is not very intuitive to operator, because the master movements do not correspond directly with the excavators movements.

The second device used was the master device of the Grips System of the Kraft Telerobotics Inc. (Figure 6). The Grips system is the master - slave, with six articular degrees of freedom slave. The master is a articular device too, with a six degrees of freedom, three for position ( $x, y$ and $z$ ) and three for orientation (roll, pitch and yaw). It can exert force only in the first five axes, with maximum load of $30 \mathrm{~N}$. The communications between master-slave is close. Due to this, the implementation in others systems are difficult, however its utilisation for excavator teleoperation carries out good results.

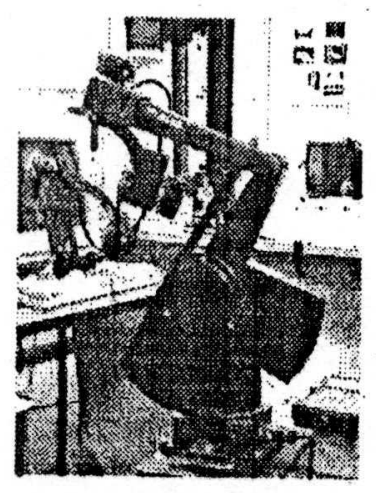

Figure 6. Master of Kraft Telerobotics

Finally, the last master used was the PHANToM system produced by Sensable Tech (Figure 7). It's a haptic device, which permits "feel" tactil sensations to operator. It provides six articular degrees of freedom, three for position $(x, y$ and $z)$ and three for orientation (roll, pitch and yaw), but in contrast with the Cybernet and Kraft systems it permits only force reflection in the first three axis. Due to its interface with the user is a thimble or stylus the teleoperation is not intuitive, additionally the maximum force exertable is $5 \mathrm{~N}$ only.

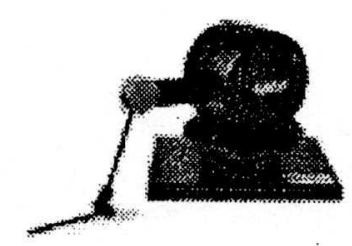

Figure 7. PHANToM master of Sensable Tech.

To implement the force feedback, it has been necessary to develop a new method to obtain the forces at the tip of the bucket of the excavator based on the incremental pressure in the chambers of the cylinders. After obtaining the force in each cylinder, the inverse Jacobian matrix is used. There have been good results without modification of the structure of the excavator (if a standard force/torque sensor would have been used, a modification of the structure would have been required with a consequence of a lack of strength and an increment in the prize). Buried obstacle detection algorithms that use these calculated forces (other developments do it with radar information ) have been developed.

Two types of bilateral control schemes have been tested: force-position, and position-position. The block diagrams of both strategies can be seen in figure 4 and figure 5 .

The key issue in a teleoperation with large reaction forces, is the force exerted on the environment. It is yery important to keep the operator informed about the force she/he is exerting to avoid potential damages in the environment, or in the excavator itself. The information provided to the operator is the video and the force. Two options appear thinking about the control schemes: force proportional to the exerted force (figure 4), or force proportional to the position error (figure 5). The former is more real, it provides a force with the same shape of the real one. The main problem is that it can produce instabilities as the feedback constant grows up. On the other side, if the constant is not large enough, the human being does not take some actions on time, like the correction of the position reference if she/he feels the contact against the terrain (more exactly, the actions are taken late, which can produce a damage in a pipe for example). [6]

If a force proportional to the tracking error, the problem of instabilities disappears but the information is not so real. In free space this issue is not so critical. In fact, the feeling is very close to force feedback when the gravity torque is subtracted. 


\section{RESULTS}

The results with both schemes have been obtained through three experiments (numbered 1 to 3 ). All the three ones consists in the accomplishment of one single dig, and the dump of the excavated soil. The master device is the Cybernet master, and the force feedback have been provided axis by axis [5]

Experiment number one implements a positionposition control. The tracking error-force gains are 0.0025 for boom and stick, and $1.510^{-4}$ for the bucket. Figure 8 and figure 9 show the results.

Experiment number two implements a force-position control with the feedback of the torques produced by the cylinders to three axes of the master device. The force gains are 1/2000 for axes number one and three (boom and bucket), and 1/1000 for axis number two (stick). Figure 10 and figure 11 show the position and force in this case.
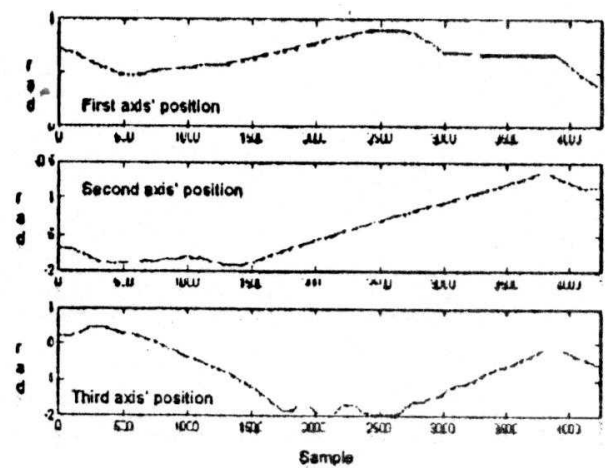

Figure 8. Positions for the experiment number one.

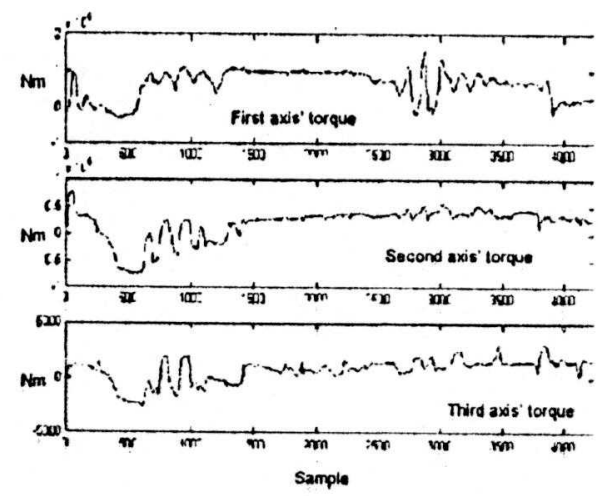

Figure 9. Torques for the experiments number one
In the last experiment, the force-position control is implemented again. The new gains are 1/3000 for boom and bucket, and 1/1200 for the stick (see figure 12 and figure 13 ).
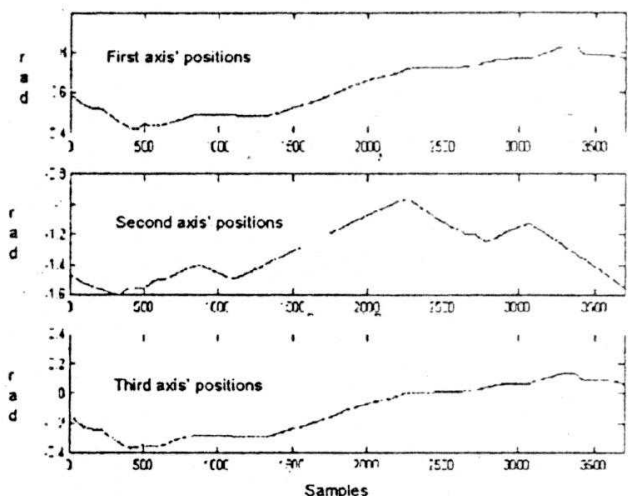

Figure 10. Positions for the experiment number two

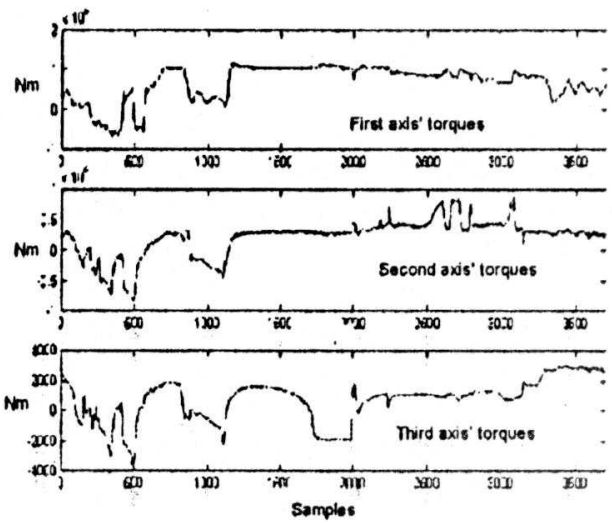

Figure 11. Torques for the experiment number two

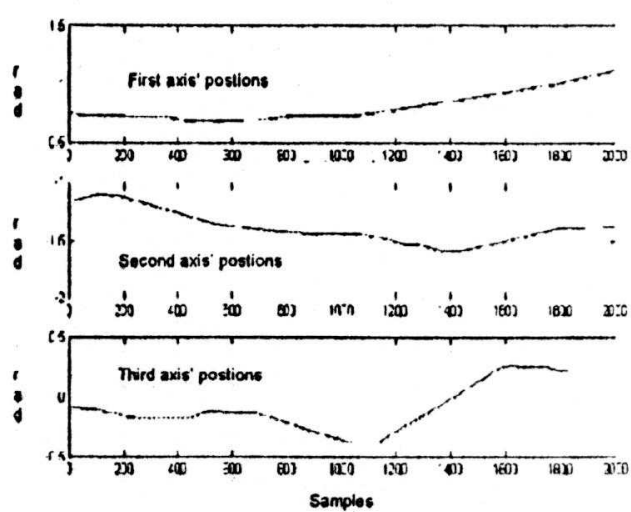

Figure 12. Positions for the experiment three 
It can be seen that with large gains in the forceposition scheme, the torques in the axis present more oscillations. In fact, the operators feel the system more uncomfortable to handle. On the other side, the position-position scheme presents fewer oscillations than in the force-position control, although the exerted forces are greater.

Rate control has been tested in free space. Basically, the masters are configured as springs (so if no force is exerted, the master moves to the equilibrium position). The opinion of the operators is that is more confortable and more accurate than the position control mode. We are currently testing the way to feedback force in this mode. One solution is the increment of the spring stiffness constant as the force exerted on the environment grows up. The other one is just the force feedback, although the main drawback is the confusion produced in the operator, because she/he does not know whether the force is due to the spring or the reaction force.
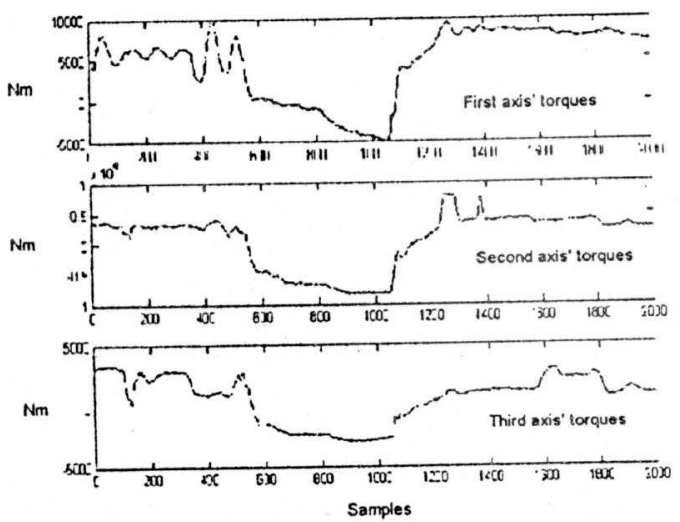

Figure 13. Torques for the experiment number three

\section{CONCLUSIONS}

The main goal of this project was the develop of a totally operative teleoperated excavator with force feedback based in one commercial. Different types of master device were tested, obtaining the best results with the articular master devices. Different bilateral control schemes like position-position and forceposition were proved. The scheme force-position demonstrated the best results.

New method to obtain the force in the tip of the bucket of the excavator war developed based in the imcasurements of the incremental pressures in the chambers of the cylinders. With the tools proportioned in this project, the next step is the implementation of the supervisory control in the teleoperation of the excavation tasks. In this way, it was developed an state predictor based on hidden Markov models.

\section{Acknowledgments}

This project was developed thanks to the sponsor of the "Comisión Internacional de Ciencia y Tecnologia" CICYT.

\section{REFERENCES}

[1] Barrientos A. et all. "The teleoperation of a Backhoe excavator through force reflection and pressure measurements" Second international workshop on service and personal robots. IARP 1997.

[2] Bradley D. A. "The development, control and operation of a autonomous robotic excavator" Journal of Intelligent and Robotics Systems, $21 \mathrm{pp}$ 75-97, 1998.

[3] Huang X. D. and Bernald L. E. "Robotic rock handling during backhoe excavation" Automation and Robotics in construction $X$. Elsevier Science Publishers 1993.

[4] Kojima Y. et all. "Remote control type excavationig robot for deep foundation" Fujikura technical review. 1990.

[5] Luengo O. "Teleoperación bilateral semiautonoma en actividades con elevadas fuerzas de reacción con el entorno" Ph.D. Tesis Universidad Politécnica de Madrid 1999. (in Spanish)

[6] Peñin L. F. "Control bilateral de robots teleoperados, Contribución en reflexión de fuerzas" Ph.D. Tesis Universidad Politécnica de Madrid 1998. (in Spanish).

[7] Singh S. "The state of the art in the automation of earth moving" ASCE journal of Aerospace Engineering. 1992.

[8] Stentz T. et all. "A robotics excavator for autonomous truck loading" Proccedings of the IEEE/RSI International conference on Intelligent robotic systems. Canada 1998.

[9] Vähä P. K. and Skibniouski M. J. " Dynamic model of an excavator" ASCE journal of Aerospace Engineering. 1993.

[10] Yoshikawa T. "Foundation on robotics" The MIT press. Cambridge Massachusetts. 1990. 